Clinical Research

\title{
Periodontitis affects glucoregulatory hormones in severely obese individuals
}

\author{
Anna Solini $\mathbb{1}^{1} \cdot$ Jean Suvan ${ }^{2} \cdot$ Eleonora Santini ${ }^{3} \cdot$ Stefano Gennai ${ }^{1} \cdot$ Marta Seghieri ${ }^{3} \cdot$ Stefano Masi ${ }^{3}$. \\ Morena Petrini ${ }^{1} \cdot$ Francesco D'Aiuto $^{2} \cdot$ Filippo Graziani $^{1}$
}

Received: 6 February 2018 / Revised: 31 August 2018 / Accepted: 26 September 2018 / Published online: 19 November 2018

(c) The Author(s) 2018. This article is published with open access

\begin{abstract}
Objective To evaluate the effect of periodontitis (PD) on glucoregulatory hormones in obesity, never explored so far, a cross-sectional study was conducted in 110 severely obese, non-diabetic individuals.

Methods We collected clinical periodontal parameters, including probing pocket depth (PPD), bleeding on probing (BOP), clinical attachment level (CAL). Insulin, glucagon, GLP-1 and GIP were measured after 3 days of standardized diet.

Results Forty-seven subjects had periodontitis (PD+) and 63 did not (PD-). PD+ showed 30.3\% of gingival sites with PPD $>4 \mathrm{~mm}, 55.2 \%$ of BOP sites and a mean CAL loss of $4.1 \mathrm{~mm}$. Compared with PD-, PD + had higher glucagon (26.60 [25.22] vs 3.93 [7.50] ng/l, $p<0.0001)$ and GIP levels (10.56 [13.30] vs 6.43 [8.43] pmol/l, $p<0.001$ ), while GLP-1 was reduced (11.78 [10.07] vs 23.34 [16.80] pmol/l, $p<0.0001)$. Insulin did not differ. In PD+, after adjustment for confounders, PPD was positively related to glucagon $(\beta=0.424, p=0.002)$ and inversely to GLP-1 $(\beta=-0.159, p=0.044)$. Conclusions We describe for the first time an impaired incretin axis coupled with a relative hyperglucagonemia in obese non-diabetic individuals with PD, that might contribute to deteriorate their glucose tolerance and partially explain the higher risk of diabetes observed in these patients.
\end{abstract}

\section{Introduction}

A bidirectional relationship links metabolic disorders like type 2 diabetes (T2D) or obesity and periodontitis (PD) [1]. The common soil between these chronic conditions is likely to be systemic, low-grade inflammation: dental plaque releases bioactive products, which in turn stimulate host responses at the gingival and systemic level, promoting the release of inflammatory cytokines like IL-1 $\beta$, IL-6, and

These authors contributed equally: Francesco D'Aiuto, Filippo Graziani

Anna Solini

anna.solini@med.unipi.it

1 Department of Surgical, Medical, Molecular and Critical Area Pathology, University of Pisa, Pisa, Italy

2 Periodontology Unit, University College London Eastman Dental Institute, London, UK

3 Department of Clinical and Experimental Medicine, University of Pisa, Pisa, Italy
TNF $\alpha$ [2, 3]. These mediators are implicated in insulin resistance, impaired $\beta$-cell function, endothelial dysfunction, driving diabetes onset and the accelerating atherosclerosis marking it. Incretin peptides, principally GLP-1 and GIP, regulate islet hormone secretion, glucose concentrations, appetite and body weight, and immune function. A significant downregulation of the incretin secretion is detected in subjects with T2D, and recent studies suggest important anti-inflammatory effects of these molecules [4]. Thus, incretins might represent a pathway potentially involved in the dysregulation of glucose metabolism in patients with PD and at high risk of T2D, and the gingival inflammation could potentially exert a detrimental action also on the incretin axis. Obesity, rather than T2D, might represent a good model to address such issue, for the relative preservation of incretin function and, on the other hand, the high prevalence of periodontal disease [5]. The present study has been designed to evaluate whether serum levels of glucoregulatory hormones and incretins might be influenced by periodontal condition in obese non-diabetic individuals. 


\section{Research design and methods}

\section{Subjects}

One hundred and ten obese (age $48 \pm 10$ years, 65 females, BMI $44.6 \pm 8.9 \mathrm{~kg} / \mathrm{m}^{2}$ ) patients were sequentially enrolled over 2015-2016 among participants in screening programs for periodontal disease. All participants signed an informed consent. Inclusion criteria were age $<60$ years, BMI $>35$ $\mathrm{kg} / \mathrm{m}^{2}$, absence of T2D (excluded by an oral glucose tolerance test, OGTT, performed 1 week before the study), no current smoking habits, no treatment with immunosuppressors/antibiotics/anti-inflammatory drugs and absence of systemic inflammatory diseases. All patients underwent a periodontal-screening visit; in those resulted positive to the periodontal screening record [6], a complete periodontal examination was performed. After 3 days of standardized $600 \mathrm{kcal}$ diet, blood samples were drawn to measure routine biochemistry, high sensitivity CRP and plasma levels of insulin, glucagon, GLP-1 and GIP.

\section{Periodontal clinical parameters}

Standard periodontal clinical parameters were registered using a UNC $15-\mathrm{mm}$ periodontal probe by a calibrated examiner as previously reported [7]. The examiner recorded full-mouth pocket probing depth (PPD) and recessions at six sites per tooth (excluding third molars). Bleeding on probing (BOP) was recorded as local bleeding present within $30 \mathrm{~s}$ upon probing. Clinical attachment level (CAL) was calculated as the sum of PPD and gingival recession. Periodontitis was defined on interproximal CAL loss and radiographic evidence of bone loss as previously reported [8]. Periodontitis was stratified according to the recommendations of the American Academy of Periodontology [9].

\section{Assays}

Plasma insulin was measured by an electrochemiluminescence assay on a COBAS e411 (Roche, Indianapolis, IN, USA). Plasma GLP-1, GIP, and glucagon were assessed using a Multiplex technique (Millipore Corp, Billerica, MA, USA and Mercodia AB, Uppsala, SW). Circulating levels of IL-6, IL-1 $\beta$, and TNF $\alpha$ were assessed by commercial ELISA kits (R\&D Systems, Minneapolis, MN, USA) following manufacturer's instructions.

\section{Statistics}

Data were reported as mean $\pm \mathrm{SD}$, or median [IQR]. Differences between groups were analysed using ANOVA or Wilcoxon rank sum test for non-normally distributed
Table 1 Clinical characteristics and circulating cytokine levels of the study participants

\begin{tabular}{|c|c|c|c|}
\hline & $\begin{array}{l}\mathrm{PD}+(n= \\
47)\end{array}$ & $\begin{array}{l}\mathrm{PD}-(n= \\
63)\end{array}$ & $p$ value \\
\hline Age (years) & $48 \pm 7$ & $48 \pm 12$ & 0.910 \\
\hline $\operatorname{Sex}(\mathrm{F} / \mathrm{M})$ & $28 / 19$ & $37 / 26$ & 0.950 \\
\hline $\mathrm{SBP}(\mathrm{mmHg})$ & $130.6 \pm 18.9$ & $130.1 \pm 16.1$ & 0.896 \\
\hline $\mathrm{DBP}(\mathrm{mm})$ & $85.9 \pm 10.6$ & $83.1 \pm 11.0$ & 0.245 \\
\hline BMI $\left(\mathrm{kg} / \mathrm{m}^{2}\right)$ & $41.4 \pm 8.7$ & $47.1 \pm 8.3$ & 0.001 \\
\hline Waist (cm) & $116.1 \pm 10.3$ & $132.1 \pm 11.7$ & 0.001 \\
\hline $\begin{array}{l}\text { Fasting plasma glucose } \\
(\mathrm{mmol} / \mathrm{l})\end{array}$ & $5.37 \pm 1.29$ & $5.09 \pm 1.23$ & 0.100 \\
\hline $\begin{array}{l}\text { Fasting plasma insulin } \\
(\mathrm{pmol} / \mathrm{l})\end{array}$ & $\begin{array}{l}90.29 \\
{[49.27]}\end{array}$ & $\begin{array}{l}80.97 \\
{[91.33]}\end{array}$ & 0.075 \\
\hline HOMA-IR & $3.38[2.41]$ & $2.71[3.58]$ & 0.05 \\
\hline Total cholesterol (mmol/l) & $5.29 \pm 1.13$ & $5.02 \pm 0.96$ & 0.182 \\
\hline Triglycerides (mmol/l) & $1.64 \pm 0.83$ & $1.61 \pm 0.80$ & 0.850 \\
\hline HDL cholesterol (mmol/l) & $1.21 \pm 0.35$ & $1.26 \pm 0.36$ & 0.473 \\
\hline Metabolic syndrome $^{\mathrm{a}}(\%)$ & 52 & 48 & 0.932 \\
\hline hs CPR (mg/l) & $4.31 \pm 3.80$ & $3.67 \pm 2.62$ & 0.290 \\
\hline IL-6 (pg/ml) & $4.45[2.10]$ & $1.99[1.34]$ & $<0.000$ \\
\hline $\mathrm{IL}-1 \beta(\mathrm{pg} / \mathrm{ml})$ & $3.53[3.32]$ & $1.99[1.88]$ & 0.002 \\
\hline $\mathrm{TNF} \alpha(\mathrm{pg} / \mathrm{ml})$ & $2.82[2.03]$ & $2.03[1.27]$ & 0.003 \\
\hline
\end{tabular}

${ }^{a}$ According to the IDF Consensus Worldwide Definition of the Metabolic Syndrome (2005)

Data are mean $\pm \mathrm{SD}$, or median[IQR]

variables. Relationships between variables were assessed using Spearman's correlation analysis and multiple linear regression analysis, including stepwise model. Statistics were performed using $\mathrm{JMP}^{\circledR} 7.0$; a $p$ value $\leq 0.05$ was considered significant.

\section{Results}

Participants were grouped based on the presence (PD,$+ n$ $=47$ ) or absence (PD-, $n=63)$ of periodontitis. Clinical characteristics of the study participants are shown in Table 1. The two groups were balanced for age and gender, while BMI was significantly higher in PD - than and PD+ individuals. Percentage of former smokers was similar between the groups. Nearly half the population in both groups had the phenotype of metabolic syndrome. Among biochemical variables, no differences emerged for fasting plasma glucose and lipid profile, whereas hsCRP resulted elevated in both groups, but no differences between groups were observed. PD+ individuals had a mean number of 26 teeth, $30.3 \%$ of gingival sites with PPD $>4 \mathrm{~mm}$, a mean $55.2 \%$ of BOP sites and an average CAL loss of $4.1 \mathrm{~mm}$, indicating a generalized level of tissue loss. 

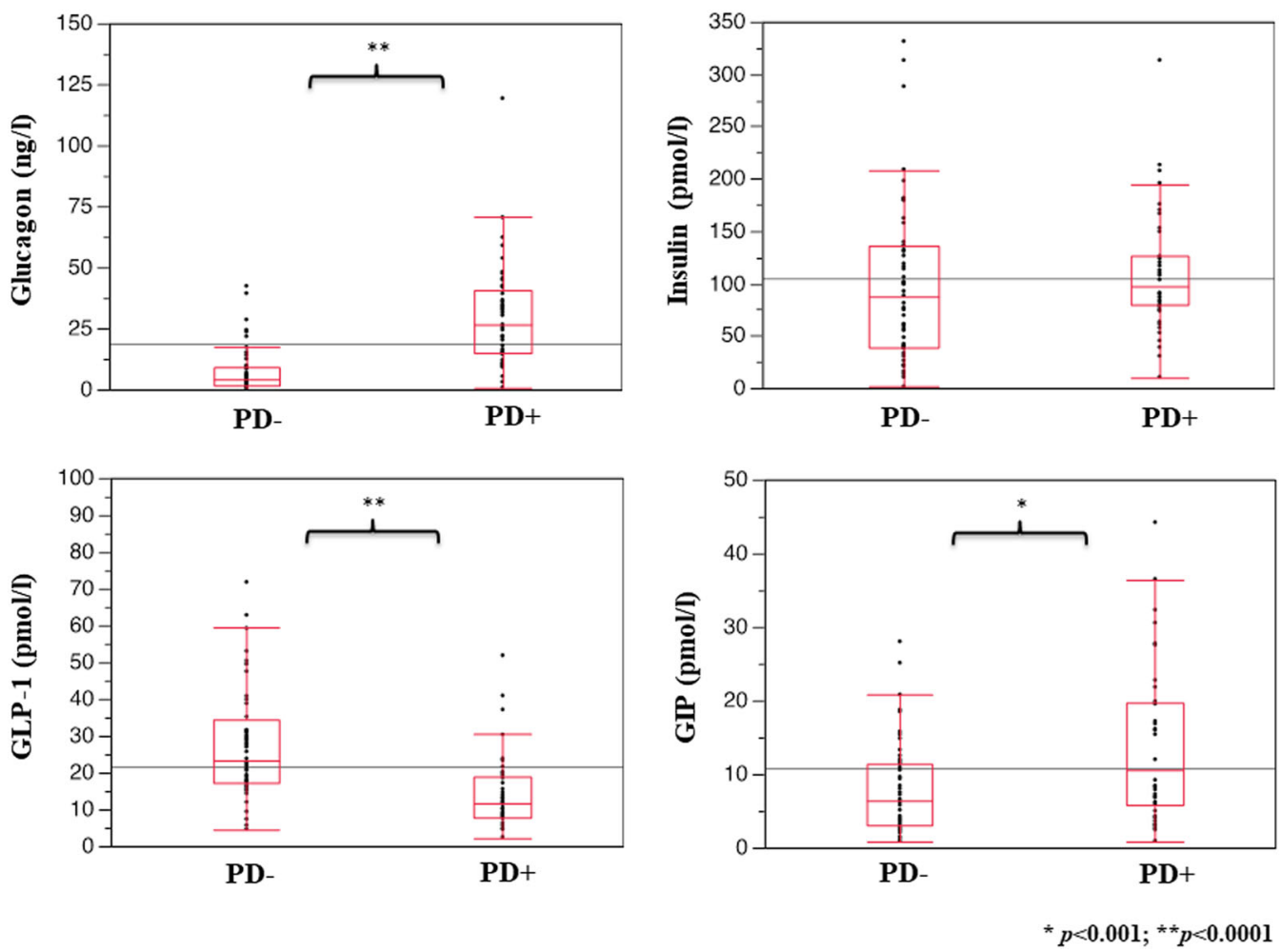

Fig. 1 Plasma levels of glucagon, insulin, GLP-1, and GIP in obese non-diabetic individuals without (PD-) and with (PD+) chronic periodontitis. Data are reported as median and IQR

Glucoregulatory hormones are shown in Fig. 1. Plasma glucagon concentrations were six-fold higher in PD+ than in PD- subjects, while GLP-1 levels were markedly reduced. Plasma insulin did not differ between the groups, while GIP was increased in PD+ individuals.

In the $\mathrm{PD}+$ group, CAL and PPD variables were linearly correlated with glucagon $(r=0.404, p=0.003$ and $r=$ $0.456, p<0.001$, respectively). For PPD, such relationship still held at multiple regression analysis including BMI, age, and gender $(\beta=0.431, p=0.002)$; moreover, when adding the incretin concentrations in the model, both glucagon $(\beta$ $=0.424, p=0.002)$ and GLP-1 $(\beta=-0.159, p=0.044)$ resulted associated (full model $r^{2}=0.30$ ). After a stepwise approach results remained almost unchanged, with glucagon, GLP-1, and BMI explaining about the total variance of PPD. When comparing different stages of PD [9], glucagon levels were related with the severity of periodontitis (16.17 $\pm 9.95,25.25 \pm 4.34,39.24 \pm 4.45 \mathrm{ng} / \mathrm{l}$ from stages I to III; $p=0.03)$.

To better detect the relation between presence of periodontitis and systemic subclinical inflammation, we measured circulating levels of some inflammatory cytokines. As reported in Table 1, IL-6, IL-1 $\beta$, and TNF $\alpha$ were significantly higher in the PD+ group. After modelling data by a multiple regression analysis, adjusting for sex, BMI, age and presence of PD, only IL-6 was significantly associated with the presence of $\operatorname{PD}(\beta=0.88, p=0.04)$.

\section{Conclusions}

This study reports for the first time a strong impairment of incretin axis in obese individuals with severe periodontitis. An upregulation of glucagon, GLP-1, and GIP has been previously described in the gingival crevicular fluid of T2D subjects with periodontitis [10], while circulating levels of these hormones have never been related to the state of the gingivae. Our findings support the notion of a temporal association between periodontitis and obesity precedent to a derangement of glucose metabolism (normal OGTT and no difference in fasting glucose levels between the two groups), and eventually driving an increased risk of T2D in obese patients affected by periodontitis. This is further corroborated by the lower profiles of common risk factors of future T2D (lower BMI and a trend toward higher plasma glucose). However, our design does not allow inferring whether weight loss with consequent incretins' variations may have a different impact in obese patients with or without periodontitis.

Intriguingly, CRP seems to mark a similar systemic lowgrade inflammation in the two groups, suggesting a primary 
involvement of the mouth-gut axis, with a key role of the periodontitis and the oral inflammation as the main determinant of the observed hormone variations.

GLP-1 appears significantly reduced in obese patients with periodontitis. We have attempted to account for the common confounders like the type of consumed food, able to influence gut hormones (our study subjects followed the same diet for 3 days before the assessment). It is of interest that raised GLP-1 levels have been reported in critically ill individuals with a severe systemic inflammatory status [11] or after a major surgical stress [12]; here, diminished GLP-1 levels are observed in humans following a local and delimited, rather than a systemic, inflammatory status.

Looking more in detail to the hormone pattern coupled with periodontitis, glucagon levels were found to be elevated and correlated with the severity of the disease. Increased fasting glucagon concentrations may be responsible of the more pronounced insulin resistance [13], as observed in PD+ individuals according to HOMA-IR. The slightly higher fasting plasma glucose, likely due to marked increase in glucagon levels, in association with marginally raised insulin levels may account for the increased HOMA$\mathrm{IR}$ in $\mathrm{PD}+$ group. Moreover, this finding is reinforced by the fact that inflammatory cytokines levels, potentially associated with insulin resistance, are higher in PD+ group. In this view, the increased GIP levels deserve attention too, as they are able to stimulate glucagon secretion; such effect may help to stabilize glucose levels in healthy individuals, whilst in T2D and obese subjects these may contribute to glucose intolerance [14]. The hypothesis of a role of anatomical contiguity between mouth and gut in influencing this picture is reinforced by the lack of difference in insulin levels between obese with and without periodontal disease, according to previous data in carriers of metabolic syndrome [15]. Previous experimental works have shown that acute inflammation promotes GLP-1 secretion [11, 16]; in the present study, the chronic subclinical inflammation, associated with periodontitis, particularly IL-6, seems to be linked to an impaired incretin balance, this happens despite a lower BMI in PD+ individuals. A mere speculation on the mechanism behind the raised glucagon levels could be the trophic effect exerted by IL-6 on alpha-cells [17]; regarding GIP, beside its metabolic effects, it has been recently shown as it may promote adipose tissue inflammation and cytokine transcripts in humans [18]. In summary, this study shows for the first time a dysregulation of the incretin axis in obese individuals with periodontitis; we might speculate the potential role of the oral/gut microbiota in determining this clinical condition that might accelerate the deterioration of glucose homoeostasis frequently occurring in these individuals; a role for subclinical inflammation cannot be ruled out too. If confirmed in large further studies, our findings suggest that the incretin axis might represent a novel important target to prevent the development of $\mathrm{T} 2 \mathrm{D}$ in patients with PD.

Author contributions AS developed the original idea, wrote the protocol, analysed the data and wrote the manuscript. JS, SG, and MP recruited participants and performed the study. ES performed the lab determinations. MS analysed the data and contributed to the discussion. SM contributed to the discussion. FD'A and FG supervised the study and reviewed/edited the manuscript. AS is the guarantor of this work.

\section{Compliance with ethical standards}

Conflict of interest The authors declare that they have no conflict of interest.

Open Access This article is licensed under a Creative Commons Attribution 4.0 International License, which permits use, sharing, adaptation, distribution and reproduction in any medium or format, as long as you give appropriate credit to the original author(s) and the source, provide a link to the Creative Commons license, and indicate if changes were made. The images or other third party material in this article are included in the article's Creative Commons license, unless indicated otherwise in a credit line to the material. If material is not included in the article's Creative Commons license and your intended use is not permitted by statutory regulation or exceeds the permitted use, you will need to obtain permission directly from the copyright holder. To view a copy of this license, visit http://creativecommons. org/licenses/by/4.0/.

\section{References}

1. Holmstrup P, Damgaard C, Olsen I, Klinge B, Flyvbjerg A, Nielsen $\mathrm{CH}$, et al. Comorbidity of periodontal disease: two sides of the same coin? An introduction for the clinician. J Oral Microbiol. 2017;9:1332710.

2. Mohamed HG, Idris SB, Ahmed MF, Åstrøm AN, Mustafa K, Ibrahim SO, et al. Influence of type 2 diabetes on local production of inflammatory molecules in adults with and without chronic periodontitis: a cross-sectional study. BMC Oral Health. 2015;15:86.

3. Khosravi R, Ka K, Huang T, Khalili S, Nguyen BH, Nicolau B, et al. Tumor necrosis factor- $\alpha$ and interleukin-6: potential interorgan inflammatory mediators contributing to destructive periodontal disease in obesity or metabolic syndrome. Mediat Inflamm. 2013;2013:728987.

4. Hirano T, Mori Y. Anti-atherogenic and anti-inflammatory properties of glucagon-like peptide-1, glucose-dependent insulinotropic polypepide, and dipeptidyl peptidase- 4 inhibitors in experimental animals. J Diabetes Investig. 2016;7:80-6.

5. Keller A, Rohde JF, Raymond K, Heitmann BL. Association between periodontal disease and overweight and obesity: a systematic review. J Periodontol. 2015;86:766-76.

6. Preshaw PM. Detection and diagnosis of periodontal conditions amenable to prevention. BMC Oral Health. 2015;15:S5.

7. Graziani F, Cei S, Tonetti M, Paolantonio M, Serio R, Sammartino $\mathrm{G}$, et al. Systemic inflammation following non-surgical and surgical periodontal therapy. J Clin Periodontol. 2010;37:848-54.

8. Tonetti MS, Claffey N, European Workshop in Periodontology group C. Advances in the progression of periodontitis and proposal of definitions of a periodontitis case and disease progression for use in risk factor research. Group C consensus report of the 5th 
European Workshop in Periodontology. J Clin Periodontol. 2005;32:210-3.

9. American Academy of Periodontology Task Force Report on the Update to the 1999 Classification of Periodontal Diseases and Conditions. J Periodontol 2015; 86: 835-8.

10. Mohamed HG, Idris SB, Mustafa M, Ahmed MF, Åstrøm AN, Mustafa $\mathrm{K}$, et al. Impact of chronic periodontitis on levels of glucoregulatory biomarkers in gingival crevicular fluid of adults with and without type 2 diabetes. PLoS ONE. 2015;10:e127660.

11. Kahles F, Meyer C, Möllmann J, Diebold S, Findeisen HM, Lebherz C, et al. GLP-1 secretion is increased by inflammatory stimuli in an IL-6-dependent manner, leading to hyperinsulinemia and blood glucose lowering. Diabetes. 2014;63:3221-9.

12. Lebherz C, Kahles F, Piotrowski K, Vogeser M, Foldenauer AC, Nassau K, et al. Interleukin-6 predicts inflammation-induced increase of glucagon-like peptide-1 in humans in response to cardiac surgery with association to parameters of glucose metabolism. Cardiovasc Diabetol. 2016;15:21.

13. Ferrannini E, Muscelli E, Natali A, Gabriel R, Mitrakou A, Flyvbjerg A, et al. Relationship between Insulin Sensitivity and Cardiovascular Disease Risk (RISC) Project Investigators.
Association of fasting glucagon and proinsulin concentrations with insulin resistance. Diabetologia. 2007;50:2342-7.

14. Christensen M, Vedtofte L, Holst JJ, Vilsbøll T, Knop FK. Glucose-dependent insulinotropic polypeptide: a bifunctional glucose-dependent regulator of glucagon and insulin secretion in humans. Diabetes. 2011;60:3103-9.

15. Benguigui C, Bongard V, Ruidavets JB, Chamontin B, Sixou M, Ferrières $\mathrm{J}$, et al. Metabolic syndrome, insulin resistance, and periodontitis: a cross-sectional study in a middle-aged French population. J. Clin Periodontol. 2010;37:601-8.

16. Nguyen AT, Mandard S, Dray C, Deckert V, Valet P, Besnard P, et al. Lipopolysaccharides-mediated increase in glucosestimulated insulin secretion: involvement of the GLP-1 pathway. Diabetes. 2014;63:471-82.

17. Ellingsgaard H, Ehses JA, Hammar EB, Van Lommel L, Quintens $\mathrm{R}$, Martens $\mathrm{G}$, et al. Interleukin-6 regulates pancreatic alpha-cell mass expansion. Proc Natl Acad Sci USA. 2008;105:13163-8.

18. Gögebakan Ö, Osterhoff MA, Schüler R, Pivovarova O, Kruse M, Seltmann AC, et al. GIP increases adipose tissue expression and blood levels of MCP-1 in humans and links high energy diets to inflammation: a randomised trial. Diabetologia. 2015;58:1759-68. 